\title{
PROJETO DE CONTROLE DE UM SISTEMA DE POSICIONAMENTO ANGULAR BASEADO EM MODELO IDENTIFICADO EM MALHA FECHADA
}

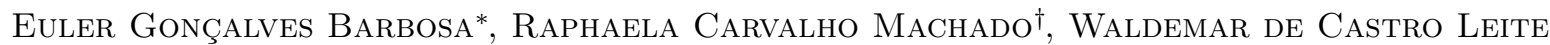 \\ FILHO $\ddagger$ \\ *Instituto de Aeronáutica e Espaço, Divisão de Sistemas Espaciais, Praça Marechal Eduardo Gomes, \\ 50, CEP 12.228-904 \\ São José dos Campos, SP, Brasil
}

Emails: euleregb@iae.cta.br, raphaelarcm@iae.cta.br, waldemarwclf@iae.cta.br

\begin{abstract}
This paper presents the identification and design of a angular positioning control system of the Quanser flexible beam which is usually used to simulate structural vibrations that occur, for example, in aircraft. The controller is designed based on the discrete model obtained from the identification of the system operating in closed loop due to its unstable nature in open loop. Initially, a subspace identification method, known as DSR_e method, is applied and designed a LQG controller based on the model to satisfy the performance requirements. Additionally, a PID controller is tuned using the multiple root locus and the golden rule is applied. The model validation is performed based on: the predictions output, MRSE index calculated from the prediction error and residual analysis. Experimental tests with the system and implementation of LQG control strategy were performed in LabVIEW environment. The results indicate that the identified model is representative of the system dynamics and were used successfully in the design of two controllers with validation on a dedicated system for real-time control.
\end{abstract}

Keywords - angular positioning control system, closed loop identification, subspace method, model-based control design.

\begin{abstract}
Resumo - Este artigo apresenta a identificação e projeto de controle de um sistema de posicionamento angular com haste flexível da QUANSER que é usualmente utilizado para simular as vibrações estruturais que ocorrem, por exemplo, em aeronaves. O controlador projetado é baseado em modelo discreto obtido a partir da identificação do sistema operando em malha fechada devido à sua natureza instável em malha aberta. Na primeira etapa do trabalho é aplicado um método de identificação por subespaços, denominado DSR_e, e em seguida, baseado no modelo, é inserido um controlador LQG para satisfazer o desempenho requerido. Adicionalmente, um controlador PID é sintonizado pela técnica do lugar geométrico das raízes múltiplo (LGR múltiplo) aplicando a regra dourada. A validação do modelo é efetuada com base: nas predições de saída, índice MRSE calculado a partir do erro de predição e análise de resíduos. Os testes experimentais com o sistema e implementação da estratégia de controle LQG foram realizados em ambiente LabVIEW. Os resultados obtidos mostram que o modelo identificado é representativo da dinâmica do sistema e foi utilizado com êxito no projeto dos controladores com validação em um sistema dedicado para controle em tempo real.
\end{abstract}

Palavras-chave - sistema de posicionamento angular, identificação em malha fechada, método de subespaços, projeto de controle baseado em modelo.

\section{Introdução}

O sistema de posicionamento angular com haste flexível da QUANSER apresentado na Figura 1 simula os movimentos de vibração de estruturas mecânicas, sendo a planta muito utilizado para validar estratégias de controle de sistema dinâmicos.

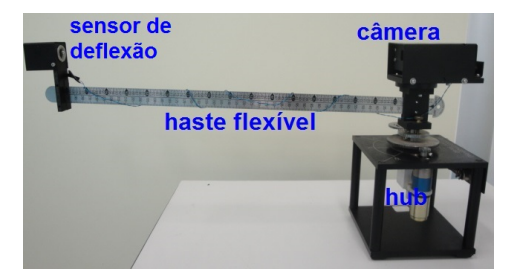

Figura 1: Sistema de haste flexível da QUANSER: cabeçote, câmera e haste flexível.

O sistema mostrado na Figura 1 consiste em um cabeçote (conhecido como hub) que rotaciona, posicionando-se angularmente mediante a aplicação de torque proveniente de um trem de engre- nagens acionado por um motor DC. Em (Garcia and Inman, 1991) são dados mais detalhes sobre o sistema.

Uma descrição matemática para o sistema é feita considerando a dinâmica da configuração mecânica do cabeçote e da estrutura flexível como movimento de corpo rígido (Barbosa and Góes, 2007). A Figura 2 apresenta as variáveis de entrada (torque $\tau$ ) e saída do sistema (ângulo de rotação do cabeçote $\theta$ e ângulo de deflexão da ponta da haste $d$ ).

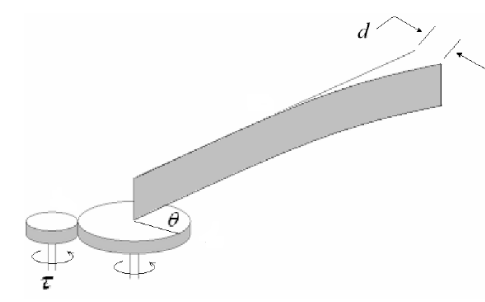

Figura 2: Engrenagens, haste flexível, torque $\tau$, deslocamento angular $\theta$ e ângulo de deflexão da ponta da haste $d$. 
Em (Távora and Ataide, 2003) é apresentada descrição matemática para obtenção do modelo do sistema, cuja representação em espaço de estados contínuo é dada por

$$
\begin{array}{r}
{\left[\begin{array}{c}
\dot{\theta} \\
\dot{d} \\
\ddot{\theta} \\
\ddot{d}
\end{array}\right]=\left[\begin{array}{cccc}
0 & 0 & 1 & 0 \\
0 & 0 & 0 & 1 \\
0 & \frac{K_{s}}{J_{b} \cdot l_{b}} & -\frac{K_{m}^{2} K_{g}^{2}}{R_{m} \cdot J_{h}} & 0 \\
0 & -\frac{K_{s}\left(J_{l}+J_{h}\right)}{J_{l} \cdot J_{h}} & -l_{b} \cdot \frac{K_{m}^{2} K_{g}^{2}}{R_{m} \cdot J_{h}} & 0
\end{array}\right]\left[\begin{array}{c}
\theta \\
d \\
\dot{\theta} \\
\dot{d}
\end{array}\right]} \\
+\left[\begin{array}{c}
0 \\
0 \\
\frac{K_{m} K_{g}}{R_{m} \cdot J_{h}} \\
-l_{b} \cdot \frac{K_{m} K_{g}}{R_{m} \cdot J_{h}}
\end{array}\right]
\end{array}
$$

em que $K_{s}(N m / r a d)$ é a constante de rigidez elástica equivalente, $K_{m}(V /(\mathrm{rad} / \mathrm{s}))$ é a constante do motor, $K_{g}$ é a razão de engrenagens, $J_{l}\left(K g m^{2}\right)$ é o momento de inércia da lâmpada, $J_{h}\left(K g m^{2}\right)$ é o momento de inércia do sensor de deflexão, $J_{b}\left(\mathrm{Kgm}^{2}\right)$ é o momento de inércia da haste flexível, $R_{m}(\Omega)$ é a resistência de armadura do motor, e $l_{b}(m)$ é o comprimento da haste flexível.

Os parâmetros do modelo em espaço de estados contínuo, considerando as constantes fornecidas no manual do fabricante e apresentadas em (Távora and Ataide, 2003), são

$$
\begin{aligned}
& A=\left[\begin{array}{cccc}
0 & 0 & 1 & 0 \\
0 & 0 & 0 & 1 \\
0 & 1199,20 & -55,4 & 0 \\
0 & -628,5 & 24,9 & 0
\end{array}\right] \\
& B=\left[\begin{array}{c}
0 \\
0 \\
103,3 \\
-46,7
\end{array}\right] \\
& C=\left[\begin{array}{llll}
1 & 1 & 0 & 0
\end{array}\right]
\end{aligned}
$$

cuja função de transferência do modelo é dada por

$$
G_{m}(s)=\frac{56,6 s^{2}-15,01 s+8921}{s\left(s^{3}+55,4 s^{2}+628,5 s+4959\right)}
$$

Neste trabalho, ao invés do uso do modelo fenomenológico, Equações 1 e 2, para o projeto de controle, considera-se a dinâmica do sistema desconhecida e a partir dos dados experimentais de entrada e saída é obtido um modelo para o sistema. O processo de identificação consiste em utilizar dados experimentais de operação em malha fechada, devido à natureza instável do sistema em malha aberta.

O trabalho de (Barbosa and Góes, 2007) apresentou a identificação do sistema apenas considerando a dinâmica do servo motor. Em (Sendrescu et al., 2008) é apresentada a identificação do sistema em malha fechada usando um método SIM.

Neste trabalho será considerada a dinâmica da haste flexível na identificação do sistema e apresentado o projeto de um controlador LQG controle baseado no modelo identificado em malha fechada também usando um método SIM.

Ênfase é dada na minimização do primeiro modo de flexão da haste. Contudo, em outras situações, cabe adotar um modelo mais representativo da dinâmica do sistema no intuito de minimizar também os modos de flexão, o que não é o escopo deste trabalho.

Nesse sentido, as principais contribuições deste artigo são:

- utilização do sistema de haste flexível da QUANSER, visto que simula as vibrações de estruturas mecânicas, como de aeronaves, o que auxilia o estudo da dinâmica de voo de sistemas;

- descrição do procedimento de identificação de um sistema real operando em malha fechada;

- aplicação de um método de subespaços para identificação de sistemas;

- projeto de um controlador digital baseado em modelo preliminarmente identificado do sistema QUANSER com viga flexível e implementação em LabVIEW da estratégia de controle tipo LQG;

- apresentação de dois projetos de controle baseado em modelo identificado com sistema operando em malha fechada, principalmente para o caso de plantas de posicionamento angular com estruturas flexíveis.

- verificação de desempenho dos controladores projetados no sentido de minimizar vibrações de estruturas mecânicas.

\section{Formulação do problema}

O processo de identificação do sistema QUANSER realizado neste trabalho consiste em utilizar dados experimentais de operação em malha fechada, cuja configuração é mostrada na Figura 3.

O algoritmo de identificação utilizado para obtenção do modelo corresponde a técnicas de subespaços que visam extrair as matrizes (parâmetros) de um modelo espaço de estados discreto para o sistema a partir da projeção de dados experimentais.

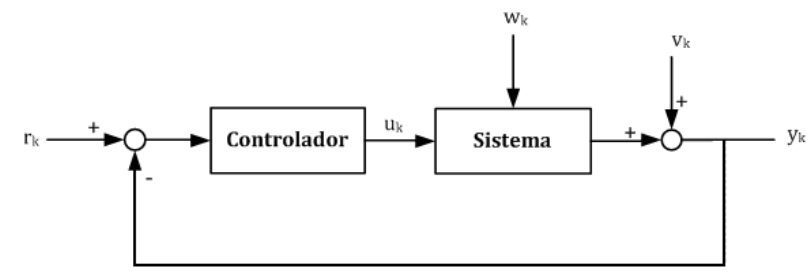

Figura 3: Configuração de um sistema em malha fechada. 
Para identificação são utilizados dados de $\left\{\mathbf{r}_{k}, \mathbf{y}_{k}\right\}$, com dinâmica do controlador conhecida. Então uma estimativa da dinâmica do sistema pode ser obtida fazendo

$$
G(s)=\frac{G_{m f}(s)}{C(s)\left[1-G_{m f}(s)\right]}
$$

Todos os ensaios realizados com o sistema QUANSER foram feitos com operação em ambiente LabVIEW, o que facilita bastante os experimentos realizados, devido às ferramentas gráficas associadas ao software. Neste trabalho o objetivo não é descrever o funcionamento do ambiente (e equipamentos) de realização dos experimentos.

\section{Procedimento de identificação do sistema em malha fechada}

Em (Katayama, 2005) é apresentado o problema de identificação por subespaços com discriminação das principais abordagens para caso de sistemas operando em malha fechada e descrição da metodologia empregada pelos métodos SIM para obtenção do modelo.

O algoritmo DSR_e (combined deterministic and stochastic system identification and realization) aplicado neste trabalho para a modelagem do sistema QUANSER é descrito em (Ruscio, 2008). O método utiliza conceitos de álgebra linear, como decomposições LQ e SVD para extrair os estados e/ou matriz de observabilidade estendida do sistema a partir de dados experimentais e determinar a ordem e parâmetros do modelo.

Para a identificação do sistema, primeiramente um controlador proporcional foi inserido na malha no intuito de estabilizar a dinâmica do sistema. O diagrama de blocos em LabVIEW é mostrado na Figura 4.

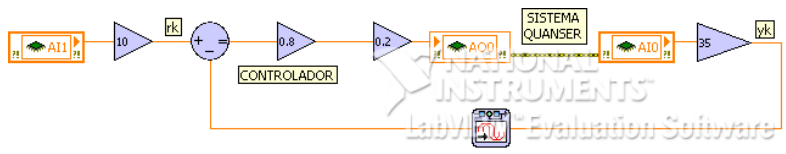

Figura 4: Sistema em malha fechada com controlador proporcional.

O sistema foi excitado durante $80 \mathrm{~s}$ e os dados foram coletados com taxa de $100 \mathrm{~Hz}$. Porém devido a grande quantidade de medições optou-se pela decimação. O novo período de amostragem é de $0,02 s$. A Figura 5 apresenta o sinal utilizado para identificação. É um sinal aleatório com patamares constantes a cada $1,5 \mathrm{~s}$, projetado baseado no tempo de resposta do sistema.

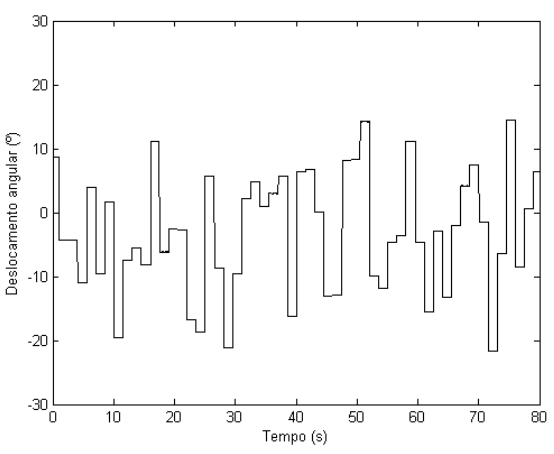

Figura 5: Sinal de referência.

Para o sinal de excitação aplicado, tem-se na Figura 6 a resposta do sistema.

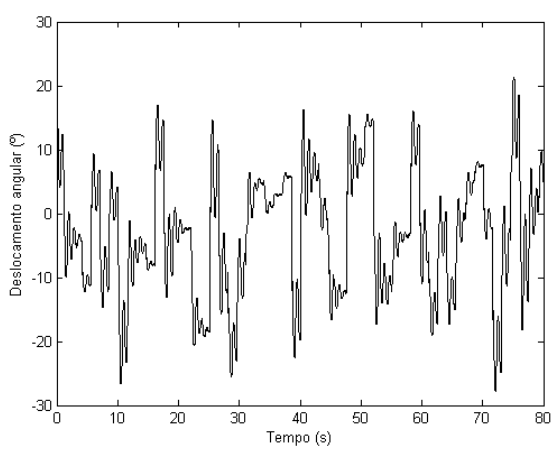

Figura 6: Sinal de saída.

Os parâmetros de identificação dos métodos de subespaços referentes à construção das matrizes bloco de Hankel dos dados experimentais passados e futuros, horizontes $J$ e $L$, respectivamente, foram adotados como $J=10$ e $L=10$. A Figura 7 apresenta a estimativa da ordem do modelo baseada nos valores singulares, sendo adotada ordem $n=4$.

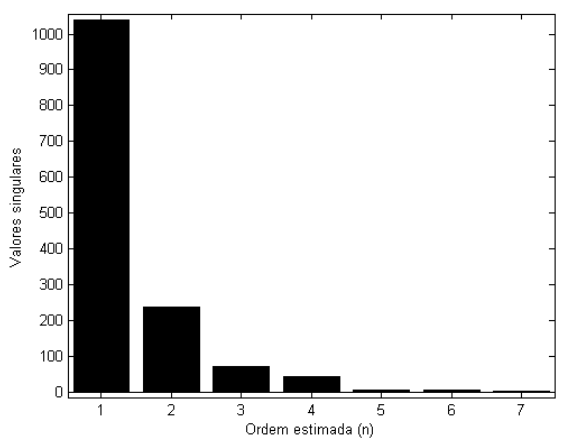

Figura 7: Estimativa da ordem do modelo baseada nos valores singulares.

Os parâmetros estimados pelo método DSR_e 
para o modelo discreto de quarta ordem são

$$
\begin{aligned}
A & =\left[\begin{array}{cccc}
0,5288 & 2,0000 & 0 & 0 \\
-0,0731 & 0,5288 & -3,4965 & -0,0983 \\
0 & 0 & 1 & -1,8698 \\
0 & 0 & 0 & 0,7552
\end{array}\right] \\
B & =\left[\begin{array}{c}
0 \\
0,0008 \\
0,0159 \\
0,4586
\end{array}\right] \\
C & =\left[\begin{array}{llll}
0,0113 & -0,1357 & -0,6543 & -0,0184
\end{array}\right]
\end{aligned}
$$

sendo $\lambda_{1,2}=0,5288 \pm j 0,3823, \lambda_{3}=0,7552 \mathrm{e}$ $\lambda_{4}=1$ os autovalores da matriz $\mathbf{A}$ e matriz $\mathbf{D}$ de transmissão direta nula.

O índice MRSE (mean relative squared error), vide (Chui and J.M., 2005), calculado para o modelo identificado pelo DSR_e é de 10,63\%, indicando baixo erro de predição. A simulação do modelo é mostrada na Figura 8, sendo a dinâmica do sistema bem capturada.

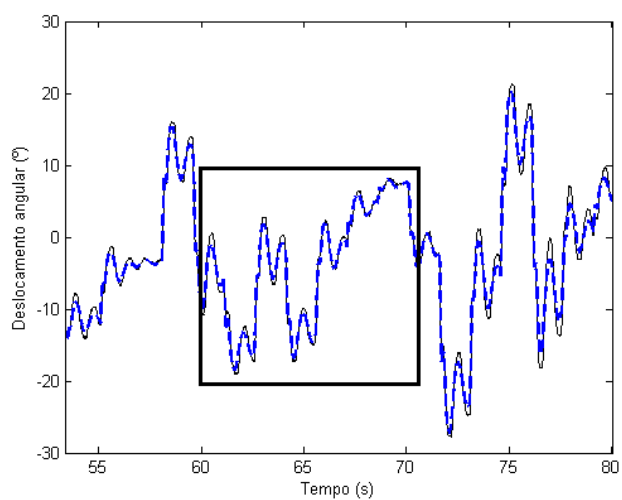

(a)

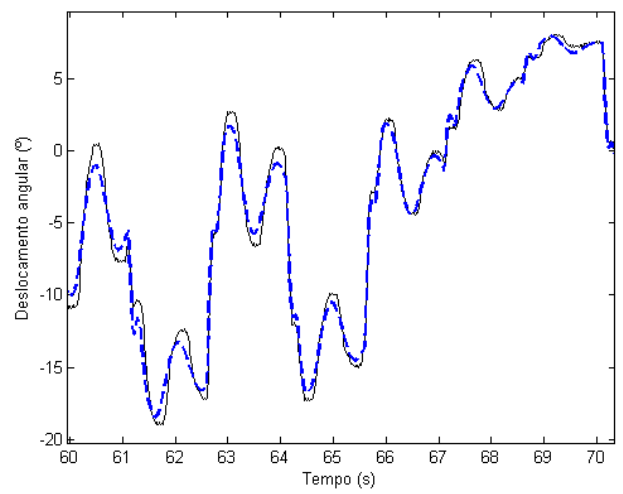

(b)

Figura 8: Predições de saída: (a) (-) dados medidos e (...) saída do modelo e (b) parte da Figura 8(a) ampliada.

Para a validação do modelo obtido, também é realizada a análise de resíduos, no intuito de verificar se o resíduo gerado pelo modelo identificado é ruído branco e os dados de entrada e o resíduo são não-correlacionados (Aguirre, 2007).

$\mathrm{Na}$ Figura 9 são apresentados os gráficos de autocorrelação do resíduo, $r_{\varepsilon \varepsilon}(\tau)$, e correlação cruzada do resíduo com a entrada, $r_{u \varepsilon}(\tau)$, para o modelo identificado pelo método DSR_e.

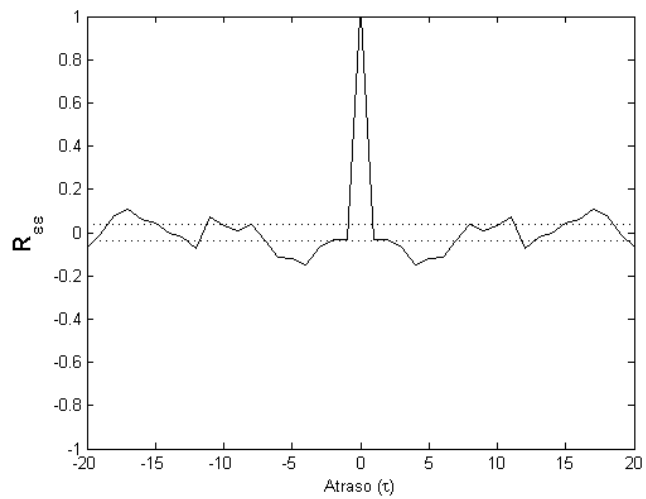

(a)

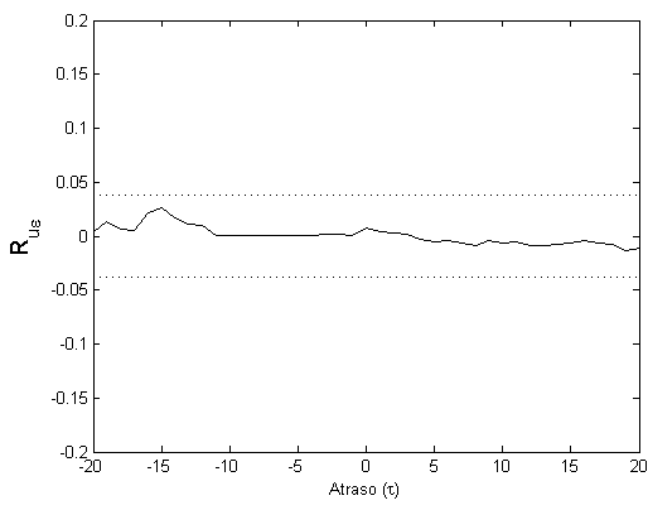

(b)

Figura 9: Análise de resíduos: (a) autocorrelação, $R_{\varepsilon \varepsilon}$ e (b) correlação cruzada, $R_{u \varepsilon}$.

A observação da Figura 9(a) sugere que o resíduo gerado pela identificação é branco, o que indica que o modelo conseguiu capturar praticamente toda a dinâmica contida nos dados. A correlação cruzada (Figura 9(b)) apresenta valores dentro do intervalo de confiança de $95 \%$ adotado.

\section{Estratégia de controle LQG}

A Figura 10 mostra a configuração da estratégia de controle LQG implementada neste trabalho, cuja formulação do problema é baseada na minimização de uma função custo quadrática do tipo

$J_{0, N}\left(U_{0, N}\right)=E\left[\sum_{k=0}^{N-1}\left\|\mathbf{Q}_{k} \mathbf{x}_{k}+\mathbf{R}_{k} \mathbf{u}_{k}+\mathbf{x}^{T} \mathbf{Q x}(N)\right\|\right]$

na qual $\mathbf{Q}_{k} \geq 0$ e $\mathbf{R}_{k}>0$ são as matrizes de ponderação dos estados e do controle, respectivamente, e o problema LQG consiste em determinar 
o ganho $\mathbf{M}_{k}$ da lei de controle (Hemerly, 2000)

$$
\mathbf{u}_{k}=\mathbf{u}_{r e f, k}-\mathbf{M}_{k}\left(\mathbf{x}_{r e f, k}-\widehat{\mathbf{x}}_{k}\right)
$$

Devido aos estados em algumas aplicações não serem medidos ou as variáveis de estados serem muito ruidosas, utiliza-se estimadores de estado. Os estados estimados $\widehat{x}_{k}$ pelo filtro de Kalman são utilizados na equação 5 e a determinação das matrizes de ponderação é feita por meio de tentativas, tal que atenda aos requisitos de desempenho desejados para o processo.

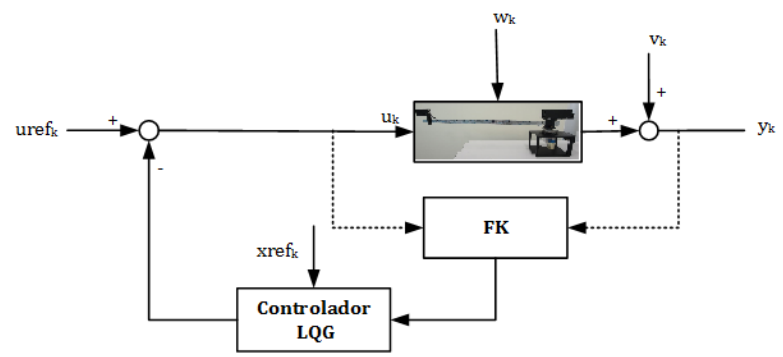

Figura 10: Esquema de um sistema em malha fechada.

O bloco Controlador LQG se refere à lei de controle dada pela equação 5 , o ganho $\mathbf{M}_{k}$ é calculado conforme descrito em (Hemerly, 2000), sendo dependente apenas das matrizes da dinâmica do sistema e das matrizes de ponderação, e o bloco FK é o estimador de estados que tem como entradas os sinais de controle e saída da planta. O estado estimado pelo filtro é utilizado para realimentar o sistema.

\section{Implementação de controladores e Resultados}

Nesta seção é descrita a implementação em tempo real do controlador LQG e também o projeto de um controlador PID baseado no modelo identificado usando a abordagem do lugar geométrico das raízes múltiplo (LGR múltiplo).

A Figura 11 apresenta o controlador LQG implementado em ambiente LabVIEW com base no modelo identificado em malha fechada, apresentado na Seção 3.

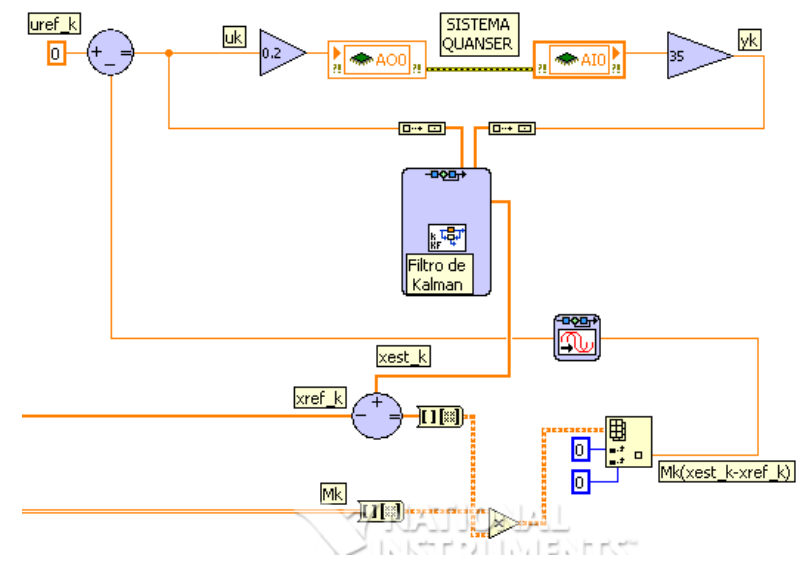

Figura 11: Diagrama de blocos em LabVIEW do sistema em malha fechada com controlador LQG.

O filtro de Kalman foi sintonizado usando variância $\sigma^{2}=0,06$ para o ruído de medição, estimada com base no sinal de saída do sistema, e desconsiderando a presença de ruído de processo.

Conclui-se pela Figura 12 que o controlador projetado fornece desempenho satisfatório para o sistema. A saída atinge o valor de referência sem sobressinal e com tempo de subida considerado favorável, baseado na dinâmica do sistema.

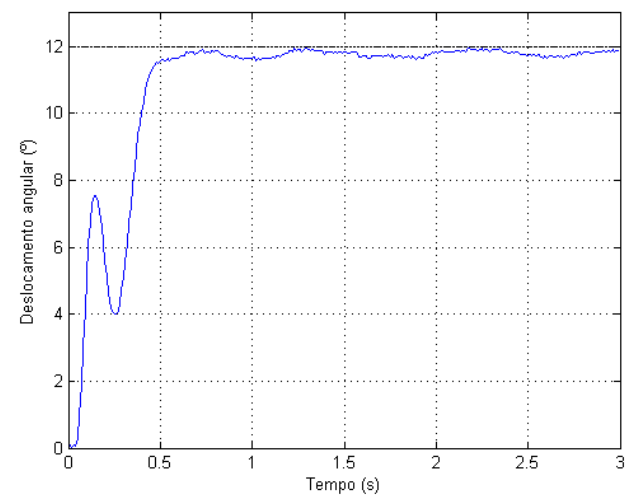

Figura 12: Resposta do sistema com controlador LQG.

Por sua vez, o projeto do controlador PID usando o LQG múltiplo consiste na análise de um conjunto de LQG's calculados com parâmetros de interesse. Para o projeto do PID considera-se a função de transferência do modelo, com algumas simplificações sendo

$$
G_{m}(s)=\frac{2230,75\left(s^{2}+3,607 s+386,6\right)}{s(s+14,04)\left(s^{2}+42,69 s+1435\right)}
$$

Assim, considera-se a planta identificada como sendo uma função de transferência com 2 zeros e 4 polos, com parâmetros $a_{n}$ e $b_{n}$ definidos conforme Equação 6, que pode ser escrita de maneira genérica como

$$
G_{\text {planta }}(s)=\frac{\sum_{n=0}^{2} a_{n} s^{n}}{\sum_{n=0}^{4} b_{n} s^{n}}
$$


A aplicação do LGR múltiplo leva ao LGR base

$$
1+k_{p} G_{\text {planta }}(s)=0
$$

A Figura 13 mostra o LGR base, com $k_{p} \in$ $[0, \infty)$, juntamente com o primeiro LGR múltiplo que é obtido com $k_{p}=\bar{k}_{p}, k_{i} \in[0, \infty)$ e $k_{d}=0$.

A equação característica para o parâmetro $k_{i} \in[0, \infty)$ é

$$
1+k_{i} \frac{\sum_{n=0}^{2} a_{n} s^{n}}{s\left(\sum_{n=0}^{4} b_{n} s^{n}+\bar{k}_{p} \sum_{n=0}^{2} a_{n} s^{n}\right)}=0
$$

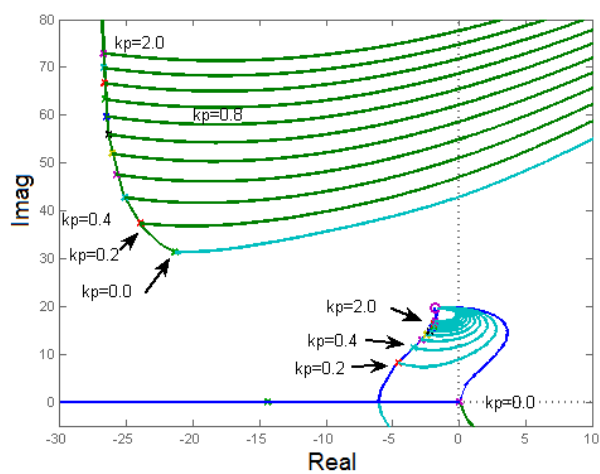

Figura 13: LGR base e primeiro LGR múltiplo $\left(k_{p}=\bar{k}_{p}, k_{i} \in[0, \infty)\right.$ e $\left.k_{d}=0\right)$.

E a equação característica para o parâmetro $k_{d} \in[0, \infty)$

$$
1+k_{d} \frac{s^{2} \sum_{n=0}^{2} a_{n} s^{n}}{\left.s\left(\bar{k}_{p} \sum_{n=0}^{2} a_{n} s^{n}+\sum_{n=0}^{4} b_{n} s^{n}\right)+\bar{k}_{i} \sum_{n=0}^{2} a_{n} s^{n}\right)}=0
$$

A escolha do parâmetro $k_{p}$ é realizada para posicionar os polos de malha fechada no semiplano esquerdo de Laplace.

A escolha de $k_{p}=0,2$ define o parâmetro constante para o cálculo dos polos de malha fechada considerando agora o parâmetro do integrador. A Figura 14 mostra os polos para $k_{i}=0,3$.

Assim, o controlador projetado com base nos parâmetros $k_{p}=0,2, k_{2}=0,3$ e $k_{d}=0,01$ foi utilizado para simulação em malha fechada do sistema de posicionamento angular da QUANSER, em que a Figura 15 apresenta a resposta ao degrau.

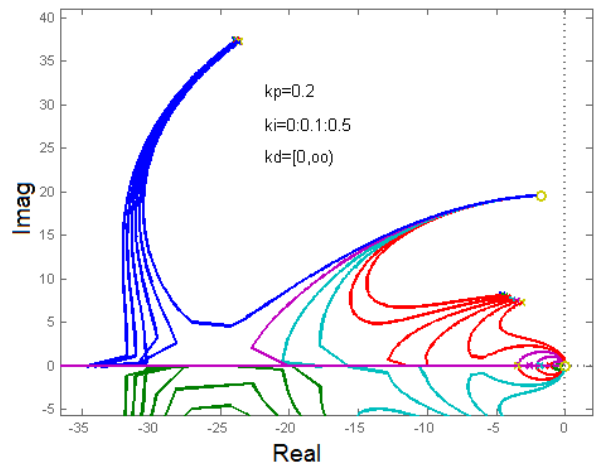

(a)

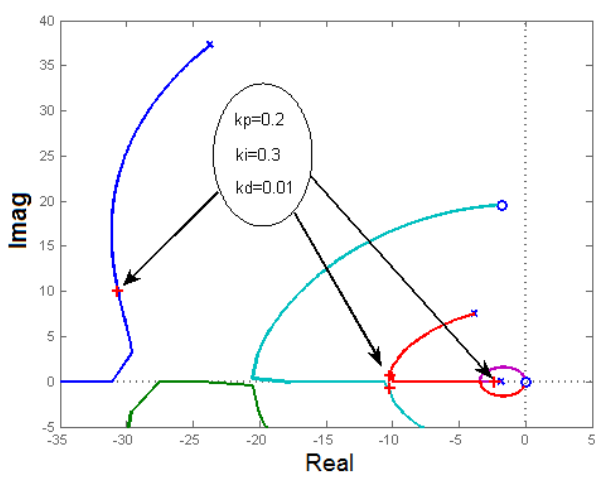

(b)

Figura 14: LGR múltiplo: (a) parâmetro do integrador, $k_{p}=\bar{k}_{p}=0,2, k_{i}=0: 0,1: 0,5 \mathrm{e}$ $k_{d} \in[0, \infty)$ e (b) parâmetro do integrador e polos de malha fechada obtios para $k_{p}=0,2, k_{i}=0,3$ e $k_{d}=0,01$.

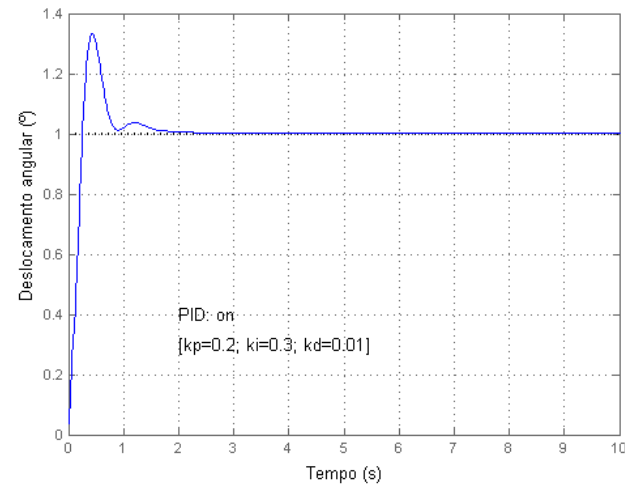

Figura 15: Resposta a degrau do sistema com controlador PID.

\section{Conclusões}

Este trabalho apresentou o projeto de controle de um sistema de posicionamento angular com haste flexível da QUANSER baseado em modelo identificado com o sistema operando em malha fechada. A planta utilizada consiste de um motor de torque e uma viga flexível que deve ser operada em ma- 
lha fechada para fins de posicionamento angular e assim, a abordagem da identificação em malha fechada para este caso é de especial interesse. Um método de subespaços foi utilizado para a identificação e o modelo obtido é representativo da dinâmica do sistema. A implementação em LabVIEW do controlador LQG projetado utilizando o modelo identificado em malha fechada foi apresentada e os resultados foram discutidos. Desse modo o presente trabalho permite a discussão de projetos de controladores para plantas com dinâmica semelhante como é o caso de foguetes de combustível líquido onde há efeitos de sloshing (chaqualhamento), pois os efeitos e as equações do movimento de uma estrutura flexível são semelhantes aos do movimento de um líquido confinado em um tanque.

\section{Agradecimentos}

Os autores agradecem ao Instituto Tecnológico de Aeronáutica e Espaço e pelo projeto SIA (Sistemas Inerciais para Aplicação Aeroespacial) pelo apoio na realização deste trabalho e em especial ao Carlos H. Silva pelo suporte técnico no laboratório do IAE, divisão ASE-C.

\section{Referências}

Aguirre, L. A. (2007). Introdução à identificação de sistemas: técnicas lineares e não-lineares aplicadas a sistemas reais., Editora UFMG, Belo Horizonte, Minas Gerais.

Barbosa, E. G. and Góes, L. C. S. (2007). Modeling and identification of flexible structure using bond graphs applied on flexcam quanser system., Congresso Brasileiro de Engenharia Mecânica-COBEM .

Chui, N. and J.M., M. (2005). Subspace identification - a markov parameter approach, International Journal of Control 78(17): 1412-1436. DOI: 10.1080/00207170500362001

Garcia, E. and Inman, D. J. (1991). Modeling of the slewing control of a flexible structure., Journal of Guidance, Control, and Dynamics 14(4): 736-742. DOI: 10.2514/3.20707

Hemerly, E. M. (2000). Controle por Computador de Sistemas Dinâmicos., Edgard Blucher.

Katayama, T. (2005). Subspace methods for system identification, Springer, Kyoto, Japan.

Ruscio, D. D. (2008). Subspace system identification of the kalman filter: open and closed loop systems., Proc. Intl. Multi-Conf. on Engineering and Technological Innovation.

Sendrescu, D., Selisteanu, D. and Marin, C. (2008). Subspace based identification of unstable systems, International Conference on
Automation, Quality and Testing, Robotics 2: 153-158.

Távora, B. G. F. and Ataide, C. A. (2003). Análise do comportamento do controle de atitude considerando esforços externos. 